\title{
Ovarian tissue cryopreservation as standard of care: what does this mean for pediatric populations?
}

\author{
Leena Nahata ${ }^{1,2} \cdot$ Teresa K. Woodruff $^{3} \cdot$ Gwendolyn P. Quinn $^{4} \cdot$ Lillian R. Meacham ${ }^{5,6} \cdot$ Diane Chen $^{7}$. \\ Leslie C. Appiah $^{6,7}$. Courtney Finlayson ${ }^{3,8}$ - Kyle E. Orwig ${ }^{9}$ - Monica M. Laronda ${ }^{10}$ • Erin E. Rowell ${ }^{3,11}$. \\ Antoinette Anazodo ${ }^{12,13}$. Olivia Frias ${ }^{14}$. Julie Sroga Rios ${ }^{14,15}$. Stacy Whiteside ${ }^{16}$. Veronica Gomez-Lobo ${ }^{17}$. \\ Maggie Dwiggins ${ }^{18}$. Krista J. Childress ${ }^{19,20}$ - Holly R. Hoefgen ${ }^{21}$. Jennifer M. Levine ${ }^{22}$. Yasmin Jayasinghe ${ }^{23}$. \\ Molly Moravek ${ }^{24}$
}

Published online: 11 May 2020

(C) Springer Science+Business Media, LLC, part of Springer Nature 2020

With steadily improving survival rates for childhood cancer and other pediatric medical conditions, it has become increasingly important to minimize anticipated adverse effects of therapies and optimize long-term quality of life [1]. Reproductive dysfunction, including infertility, is a late effect of some therapies and infertility distress is often linked to psychosocial distress among female cancer survivors $[2,3]$. For these reasons, it is critical to offer options to protect and preserve fertility prior to initiating gonadotoxic therapies [1].

Until recently, standard female fertility preservation (FP) options (i.e., oocyte and embryo cryopreservation) have only been available to post-pubertal individuals. Ovarian tissue cryopreservation (OTC) emerged as an experimental option to freeze ovarian tissue and is currently the only pre-treatment FP option for pre-pubertal children and for patients who cannot delay life-saving therapy [4]. Invasiveness of procedures, inability to delay treatment, and high costs are often barriers to standard FP, particularly for adolescents [5]. The suitability of an ovary as a transplantable organ dates back to surgeon Robert Morris, practicing in the early twentieth century, where he used this particular surgery as a "cure" for problems such as amenorrhea and infertility [6]. The first evidence that ovarian follicles could be cryopreserved and successfully complete all aspects of folliculogenesis and gamete maturation was accomplished in a mouse model by Gosden and colleagues in the late twentieth century [7]. This was followed by groundbreaking work in sheep several years later [8] and then finally, fresh and frozen auto-transplanted human tissue today $[9,10]$.

Leena Nahata

leena.nahata@nationwidechildrens.org

Extended author information available on the last page of the article
With a steadily increasing number of live births reported worldwide from cryopreserved mature ovarian tissue [10], many clinicians and researchers have questioned whether OTC should still be viewed as experimental [11]. Based on cumulative evidence, the most recent guideline published by the American Society for Reproductive Medicine (ASRM) states that OTC is an "acceptable fertility preservation technique and is no longer considered experimental" [12]. This label change will certainly have implications on the field, particularly for pediatrics.

The Pediatric Initiative Network (PIN) of the Oncofertility Consortium is a global multidisciplinary group of specialists in oncology, endocrinology, gynecology, reproductive endocrinology, urology, and psychology [13]. As leaders of the PIN, we believe we should highlight some important considerations for pediatric fertility preservation specialists in the context of this new guideline. Specifically, (1) providers must consider if and how this label change may alter approaches to counseling pediatric patients at risk for infertility and their families about fertility preservation; (2) existing OTC IRB protocols might need to be modified or eliminated; yet, OTC research needs to continue; and (3) clinicians and researchers should also consider unique implications of this new guideline for non-oncologic populations who are also at risk for future infertility.

\section{Approaches to counseling}

Timely counseling and provider recommendations have an important influence on fertility preservation attempts among pediatric patients with cancer $[13,14]$. The major topics that need to be addressed include infertility risk, fertility preservation options based on pubertal stage, risks/benefits of those 
options from both a medical and ethical perspective, in addition to costs and logistical constraints [13, 15]. Although OTC is no longer considered experimental, as the ASRM guideline notes [12], it is important to acknowledge the limited evidence, to date, of live births from ovarian tissue harvested in pediatric populations. Specifically, although there have been $>130$ live births worldwide from cryopreserved ovaries, only one of these has been from a prepubertal ovary with a second from a patient who was postpubertal but premenarcheal $[10$, $12,16,17]$. Thus, families of prepubertal children should be advised that ovary retrieval for the purposes of OTC is a relatively low-risk procedure for the patient but the future benefits in this population are less clear [18]. In this context, removal of an ovary for OTC should primarily be considered in pediatric patients who are at significant risk for infertility. Further, in more complex cases, guidance from a clinical ethics response team may assist providers in counseling patients and families in decision making [15]. Beyond the potential physical risks of fertility preservation procedures, the financial burden of the procedures (generally $>\$ 1000$ ) and long-term storage must always be considered. With increasing efforts to mandate insurance coverage for fertility preservation for medical indications, accepting OTC as standard of care may hopefully expand access and change approaches to billing at many institutions.

\section{Research implications}

Previous guidelines published by ASRM and American Society of Clinical Oncology have recognized tissue cryopreservation as investigational, to be implemented in the context of an IRB-approved protocol. Some IRBs have challenged principal investigators to identify a specific research question, particularly if all of the tissue is stored for clinical use and no data are being collected. One important implication of removal of the experimental label from OTC could be the need to modify or eliminate existing IRB protocols. However, we advocate continuing IRB-approved research studies in order to address many fundamental scientific and clinical questions which remain unanswered with regard to OTC in pediatrics. These studies should address knowledge gaps in ovarian tissue development through the pubertal transition, pediatric oocyte quality, success rates of future pediatric ovarian tissue transplantation (assessed through oncofertility registries), timeliness of pediatric fertility preservation counseling and procedures, as well as patient/family satisfaction. The PIN Research Committee aims to establish a national OTC database and centralized tissue research centers, with the goal of collaborating with international centers in order to achieve adequate sample sizes to address some of these important research questions. Thus, rather than eliminating IRB protocols entirely, we see this label change on OTC as an opportunity for oncofertility specialists to engage in collaborative, multi-site longitudinal research efforts to move the field forward.

\section{Non-oncologic populations}

As the field of "oncofertility" has progressed, awareness has grown about other pediatric populations at risk for infertility. Some of these populations are at risk due to gonadotoxic therapies for rheumatologic/renal diseases managed with lowerdose alkylating agents; sickle cell disease which may be treated with bone marrow transplant; and gender dysphoria treated with medical and/or surgical interventions that may impact future fertility [13]. In addition to treatment-related infertility, gonadal function is impaired in some pediatric populations due to the underlying genetic conditions, such as Turner syndrome and galactosemia [13]. Given the significant knowledge gaps with regard to impact of some of these conditions/ treatments on long-term gonadal function and future reproductive potential of the cryopreserved tissue, we would question whether OTC should indeed be offered as "standard of care" to all of these populations. Rather, risk/benefit counseling based on available evidence relevant to the specific patient should be provided. Additionally, research should be conducted to inform fertility and reproductive health practices in these populations.

\section{Conclusion}

Overall, we celebrate moving OTC to standard of care as a valuable step to expand the fertility preservation option toolbox. However, much remains unknown about the risks and benefits of OTC in the pediatric population, particularly in young children and non-oncologic populations. We advocate for expanded access to OTC for all populations at significant risk for infertility; nevertheless, careful counseling about the limitations of our knowledge and ongoing research in these less studied populations remains essential.

\section{References}

1. Suh E, Stratton KL, Leisenring WM, Nathan PC, Ford JS, Freyer DR, et al. Late mortality and chronic health conditions in long-term survivors of early-adolescent and young adult cancers: a retrospective cohort analysis from the childhood cancer survivor study. Lancet Oncol. 2020;21(3):421-35.

2. Lehmann V, Keim MC, Nahata L, Shultz EL, Klosky JL, Tuinman MA, et al. Fertility-related knowledge and reproductive goals in childhood cancer survivors: short communication. Hum Reprod Oxf Engl. 2017;32(11):2250-3. 
3. Armuand GM, Wettergren L, Rodriguez-Wallberg KA, Lampic C. Desire for children, difficulties achieving a pregnancy, and infertility distress 3 to 7 years after cancer diagnosis. Support Care Cancer Off J Multinatl Assoc Support Care Cancer. 2014;22(10):2805-12.

4. Wallace WHB, Anderson RA, Irvine DS. Fertility preservation for young patients with cancer: who is at risk and what can be offered? Lancet Oncol. 2005;6(4):209-18.

5. Quinn GP, Vadaparampil ST, Gwede CK, Miree C, King LM, Clayton HB, et al. Discussion of fertility preservation with newly diagnosed patients: oncologists' views. J Cancer Surviv Res Pract. 2007;1(2):146-55.

6. Gosden RG, Robert T, Morris MD. Appreciation of an enlightened surgeon and pioneer of ovarian transplantation. Fertil Steril. 2010;94(6):1960-3.

7. Carroll J, Whittingham DG, Wood MJ, Telfer E, Gosden RG. Extraovarian production of mature viable mouse oocytes from frozen primary follicles. J Reprod Fertil. 1990;90(1):321-7.

8. Gosden RG, Baird DT, Wade JC, Webb R. Restoration of fertility to oophorectomized sheep by ovarian autografts stored at -196 degrees C. Hum Reprod Oxf Engl. 1994;9(4):597-603.

9. Silber S, Kagawa N, Kuwayama M, Gosden R. Duration of fertility after fresh and frozen ovary transplantation. Fertil Steril. 2010;94(6):2191-6.

10. Donnez J, Dolmans M-M. Fertility preservation in women. N Engl J Med. 2017;377(17):1657-65.

11. von Wolff M, Sänger N, Liebenthron J. Is ovarian tissue cryopreservation and transplantation still experimental? It is a matter of female age and type of cancer. J Clin Oncol Off J Am Soc Clin Oncol. 2018:JCO1800425.

12. Ethics Committee of the American Society for Reproductive Medicine. Electronic address: ASRM@asrm.org. Fertility preservation and reproduction in patients facing gonadotoxic therapies: an ethics committee opinion. Fertil Steril 2018;110(3):380-6.

13. Moravek MB, Appiah LC, Anazodo A, Burns KC, Gomez-Lobo V, Hoefgen HR, et al. Development of a pediatric fertility preservation program: a report from the pediatric initiative network of the oncofertility consortium. J Adolesc Health Off Publ Soc Adolesc Med. 2019;64(5):563-73.

14. Lewin J, Ma JMZ, Mitchell L, Tam S, Puri N, Stephens D, et al. The positive effect of a dedicated adolescent and young adult fertility program on the rates of documentation of therapy-associated infertility risk and fertility preservation options. Support Care Cancer Off J Multinatl Assoc Support Care Cancer. 2017;25(6):1915-22.

15. McDougall RJ, Gillam L, Delany C, Jayasinghe Y. Ethics of fertility preservation for prepubertal children: should clinicians offer procedures where efficacy is largely unproven? J Med Ethics. 2018;44(1):27-31.

16. Demeestere I, Simon P, Dedeken L, Moffa F, Tsépélidis S, Brachet C, et al. Live birth after autograft of ovarian tissue cryopreserved during childhood. Hum Reprod Oxf Engl. 2015;30(9):2107-9.

17. Matthews SJ, Picton H, Ernst E, Andersen CY. Successful pregnancy in a woman previously suffering from $\beta$-thalassemia following transplantation of ovarian tissue cryopreserved before puberty. Minerva Ginecol. 2018;70(4):432-5.

18. Rowell EE, Corkum KS, Lautz TB, Laronda MM, Walz AL, Madonna MB, et al. Laparoscopic unilateral oophorectomy for ovarian tissue cryopreservation in children. J Pediatr Surg. 2019;54(3):543-9.

Publisher's note Springer Nature remains neutral with regard to jurisdictional claims in published maps and institutional affiliations.

\section{Affiliations}

\section{Leena Nahata $^{1,2}$ - Teresa K. Woodruff ${ }^{3} \cdot$ Gwendolyn P. Quinn $^{4} \cdot$ Lillian R. Meacham $^{5,6} \cdot$ Diane $^{\text {Chen }}{ }^{7}$. Leslie C. Appiah $^{6,7}$ • Courtney Finlayson ${ }^{3,8} \cdot$ Kyle E. Orwig $^{9}$ • Monica M. Laronda ${ }^{10}$ • Erin E. Rowell ${ }^{3,11}$. Antoinette Anazodo ${ }^{12,13}$. Olivia Frias ${ }^{14}$ • Julie Sroga Rios ${ }^{14,15}$. Stacy Whiteside ${ }^{16}$. Veronica Gomez-Lobo $^{17}$. Maggie Dwiggins $^{18} \cdot$ Krista J. Childress $^{19,20} \cdot$ Holly R. Hoefgen ${ }^{21} \cdot$ Jennifer M. Levine ${ }^{22}$ Yasmin Jayasinghe $^{23}$. Molly Moravek ${ }^{24}$}

1 Abigail Wexner Research Institute, Nationwide Children's Hospital, 700 Children's Drive, Columbus, OH 43205, USA

2 Department of Pediatrics, Pediatric Endocrinology, The Ohio State University College of Medicine, Columbus, OH, USA

3 Department of Pediatrics, Northwestern University Feinberg School of Medicine, Chicago, IL, USA

4 Department of Obstetrics and Gynecology, New York University School of Medicine, New York, NY, USA

5 Aflac Cancer Center, Children's Healthcare of Atlanta, Atlanta, GA, USA

6 Department of Pediatrics, Division of Hematology/Oncology and Endocrinology, Emory University, Atlanta, GA, USA

7 Department of Psychiatry, and Behavioral Sciences, and Pediatrics, Northwestern University, Chicago, IL, USA

8 Division of Pediatric Endocrinology, Ann \& Robert H. Lurie Children's Hospital of Chicago, Chicago, IL, USA
9 Department of Obstetrics and Gynecology and Reproductive Sciences, University of Pittsburgh, Pittsburgh, PA, USA

10 Stanley Manne Children's Research Institute, Ann \& Robert H Lurie Children's Hospital of Chicago, Chicago, IL, USA

11 Department of Surgery, Ann \& Robert H. Lurie Children's Hospital of Chicago, Chicago, IL, USA

12 Kids Cancer Centre, Sydney Children's Hospital, Sydney, Australia

13 Nelune Comprehensive Cancer Centre, Prince of Wales Hospital, Sydney, Australia

14 Division of Pediatric and Adolescent Gynecology, Comprehensive Fertility Care and Preservation Program, Cincinnati Children's Hospital Medical Center, Cincinnati, OH, USA

15 Department of Obstetrics \& Gynecology, University of Cincinnati, Cincinnati, OH, USA

16 Division of Hematology/Oncology, Nationwide Children's Hospital, Columbus, OH, USA 
17 Division of Pediatric and Adolescent Gynecology, Eunice Kennedy Shriver National Institute of Child Health and Human Development, Bethesda, MD, USA

18 Pediatric and Adolescent Gynecology, Norton Children's Hospital, Louisville, KY, USA

19 Department of Gynecology and Obstetrics, Division of Gynecologic Specialties, Emory University, Atlanta, GA, USA

20 Division of Pediatric Surgery, Children's Healthcare of Atlanta, Atlanta, GA, USA
21 Division of Pediatric and Adolescent Gynecology, Washington University School of Medicine, St. Louis, MO, USA

22 Division of Pediatric Hematology/Oncology, Weill Cornell Medicine, New York, NY, USA

23 Royal Children's Hospital, Melbourne, Australia

24 Department of Obstetrics and Gynecology, Division of Reproductive Endocrinology and Infertility, University of Michigan, Ann Arbor, MI, USA 